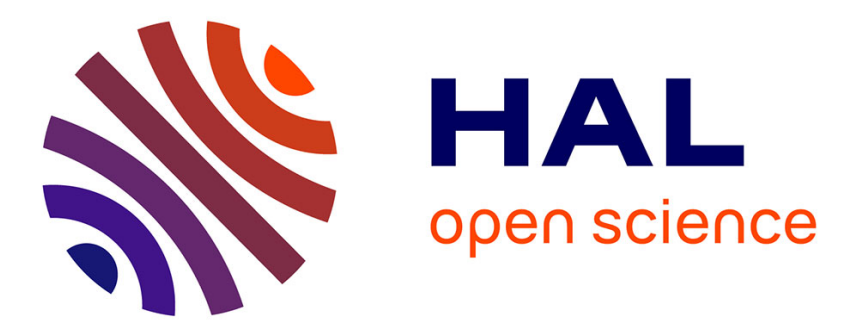

\title{
A Worked-Out Experience in Programming Humanoid Robots via the Kinetography Laban
}

\author{
Paolo Salaris, Naoko Abe, Jean-Paul Laumond
}

\section{To cite this version:}

Paolo Salaris, Naoko Abe, Jean-Paul Laumond. A Worked-Out Experience in Programming Humanoid Robots via the Kinetography Laban. Dance Notations and Robot Motion, Springer International Publishing, pp.339 - 359, 2016, 978-3-319-25737-2. 10.1007/978-3-319-25739-6_16 . hal-01376737

\section{HAL Id: hal-01376737 https://hal.science/hal-01376737}

Submitted on 5 Oct 2016

HAL is a multi-disciplinary open access archive for the deposit and dissemination of scientific research documents, whether they are published or not. The documents may come from teaching and research institutions in France or abroad, or from public or private research centers.
L'archive ouverte pluridisciplinaire $\mathbf{H A L}$, est destinée au dépôt et à la diffusion de documents scientifiques de niveau recherche, publiés ou non, émanant des établissements d'enseignement et de recherche français ou étrangers, des laboratoires publics ou privés. 


\title{
A worked-out experience in programming humanoid robots via the Kinetography Laban
}

\author{
Paolo Salaris, Naoko Abe and Jean-Paul Laumond \\ LAAS-CNRS, 7 avenue du colonel Roche, 31000, Toulouse Cedex-4, \\ e-mail: psalaris, nabe, jplelaas.fr
}

\begin{abstract}
This chapter discusses the possibility of using Laban notation to program humanoid robots. Laban notation documents human movements by a sequence of symbols that express movements as defined in the physical space. We show, by reasoning around the simple action of "taking a ball", the flexibility of the notation that is able to describe an action with different level of details, depending on the final objective of the notation. These characteristics make Laban notation suitable as a high level language and as a motion segmentation tool for humanoid robot programming and control. The main problem in robotics is to express actions that are defined and operate in the physical space in terms of robot motions that originate in the robot motor control space. This is the fundamental robotics issue of inversion. We will first show how symbols used by Laban to describe human gestures can be translated in terms of actions for the robot by using a framework called Stack of Tasks. We will then report on an experience tending to implement on a simulated humanoid platform the notation score of a "Tutting Dance" executed by a dancer. Once the whole movement has been implemented on the robot, it has been again notated by using Laban notation. The comparison between both scores shows that robot's movements are slightly different from dancer's ones. We then discuss about plausible origins of these differences.
\end{abstract}

\section{Introduction}

\subsection{Motions and Symbols}

How to transform an action expressed in the physical space (i.e. "take the ball") in terms of a sequence of motions that originate in the motor control space (i.e. "bend the legs and then move the left hand forward")? The question constitutes the essence of robotics. It opens two main challenges: motion segmentation and motion generation. The segmentation of complex movements is a fundamental step in order to make easier programming the robot and execute a given action. However, the definition of these units of action or movement primitives remains an open problem. 
In that context, it is natural to consider dance notations as a promising route to decompose complex actions into sequences of elementary motions. ${ }^{1-3}$ Indeed, the main purpose of dance notation is to store choreographic works and knowledge of dance techniques by translating movements taking place in physical space into specific ways as abstract symbol, letters, abbreviation, musical notation, stick figure, etc. In western culture, there are almost 90 dance notation systems, from the first appearance in 15 th century to the present. ${ }^{4}$ Today, among the most popular ones, we find the Kinetography Laban, the Benesh Movement Notation system and the Eshkol-Wachman Movement Notation system (see respectivelively Chalet-Haas', Mirzabekiantz' and Drewes' Chapters in this book).

This chapter reports on an experience in programming humanoid robots via the Kinetography Laban notation system.

The scope of Kinetography Laban is more general than only dance area. It aims at scoring all human motions independently of any behavior or any action. The system makes use of three types of movement symbols addressing respectively the direction of the movement, the part of the body doing the movement, the duration of the movement. It may be completed by a so-called "effort" symbol describing the dynamic quality of the movement (see Loureiro de Souza' Chapter on Laban Movement Analyzis).

\subsection{Action versus motion segmentations. Physical versus control spaces}

All dance notation systems aim at describing the motions of the body as they are observed by human eyes. The purpose is to segment and to annotate the motions of body parts as expressed in the space (e.g. "the right hand is moving slowly forward"). The scoring operates in the physical space. A dancer who has been trained to dance notation is able to embody motion symbols: When reading a score, he/she "sees" the movement of the right hand in the physical space, and he/she "knows" what to do to move it slowly forward. Muscle activation is implicit. It is performed without explicit awareness of his/her muscle control space.

In robotics and in particular in humanoid robots, the segmentation of complex movements is a more complex issue. The robot has to obey a command given by an operator (e.g. "take the ball"). The command is expressed in the physical space as an action to be performed. The first difficulty is to express the command in terms of a sub-task sequence (e.g. "to take the ball, the robot has first to go to the ball and then to grasp the ball"). Task are then translate in terms of motion units (e.g. according to the context "take the ball" may mean "move the right hand forward"). Both the decomposition of an action into sub-tasks to be performed, and the translation of each sub-task in terms of motion units, constitute the first issue to be considered. The second issue is the translation of the motions expressed in the physical space into motions expressed in the control space. A robot does not a priori "know" what to do to move its hand forward. Differently from dance notations that take into 


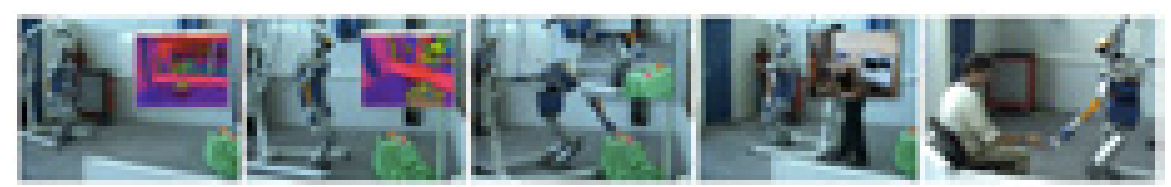

Fig. 1: The action "give Florent the ball" is decomposed into a sequence of elementary tasks.

account only the physical space around the body where actions are defined and operate, the issue of translating these actions, expressed in the physical space, into motions expressed in the motor control space, is fundamental in robotics.

Indeed, only once suitable control inputs are defined in the motor control space the robots can execute a given action in the physical space. However, the question of the segmentation is double. It deals with both action and motion decomposition:

- A given action may require a reasoning to decompose it into a sequence of subtasks to be performed. This is the task planning issue. ${ }^{5}$ Fig. 1 illustrates such a decomposition. To give Florent the ball, HRP2 robot has to locate the ball, to go to the ball, to take the ball, to locate Florent, to go to Florent, and finally to give Florent the ball. Elementary tasks as go to, take and give, require motion generation. ${ }^{6}$ On the other hand, motion planning for robots manipulating movable objects among obstacles gives rise to decomposition issues. ${ }^{7}$ The solution consists in structuring the configuration space of both the robot and the object into two elementary sub-spaces: the "grasping space" and the "placement space". The topological structure of such sub-spaces directly reflects all possible segmentation of manipulation problems. The manipulation plan appears now as a sequence of motions lying in different sub-space that embed a natural decomposition of the problem, i.e. a natural segmentation that solves the manipulation planning. Task planning in such contexts is out of the scope of the current chapter.

- As motion segmentation is concerned, it is important to consider the clear distinction between physical space and motor control space. While dance notations operate movement segmentations in the physical space, robot programming requires to operate in the motor control space. The segmentation of a movement in the physical space does not necessarily imply the same segmentation in the motor control space. For particular cyclic or repetitive actions, as e.g. elliptical and figure eight patterns of different sizes and orientations performed by using the whole arm, there is no evident segmentation in the motor control space ${ }^{8}$ but rather continuous oscillatory patterns.

Coming back to Kinetography Laban, a Laban score represents by symbols which parts of the body, e.g. arms or legs, should move and where. The 27 direction symbols can be used to segment robot actions. These symbols can be translated in elementary tasks defined in the physical space. Each elementary task consists in moving a body part towards a desired direction specified by that symbol. The sequence 
of symbols then reproduces a whole action. Now the question for the roboticist is: how to translate motions defined in the physical space into motions defined in the motor control space? Indeed, Kinetography Laban is for humans and the brain can generate suitable signals to be sent to muscles in order to move arms, limbs and in general the whole body with the aim of reaching an establish final configuration. For humanoid robots the problem is much more challenging. First of all, these complex mechanical systems are usually actuated by motors instead of muscles. Each motor is in charge of moving a part of the robot with respect to another one. As a consequence, by controlling these motors it is possible to move the robot in order to make it walking, taking an object and in general executing a established actions. In robotics, a methods for controlling the motors in order to accomplish multiple tasks expressed in the physical space is the so called Stack of Tasks (SoT). ${ }^{9}$

\subsection{Experience overview}

In this chapter, we show how the SoT can be used to translate the Laban score into control signals to be sent to the motors of a humanoid robot in order to move a body part towards a direction, specified by the corresponding symbol in the Laban score.

Section 2 introduces the SoT framework which basically provides a method for real-time control of redundant manipulators and hence useful also to control humanoid robots. We show how flexible is the notation to express human movements, and how this peculiarity is very useful in programing humanoid robots. While considering the example of the simple action of taking a ball on the floor, we show that Kinetography Laban allows annotating different levels of details.

In Section 3, we show how to translate Laban scores into tasks for the humanoid robot by using SoT. In particular, we will translate the Laban score of a "Tutting Dance", which involves only upper body movements, in a hierarchical sequence of tasks in the SoT to be executed by a simulated humanoid robot ("Romeo" from Aldebaran Robotics). The final goal is to compare the Romeo's movement with the dancer's one. The main difference concerns the quality of the Romeo's movement which is not same as the movement written in the original score.

For humanoid robots the problem of moving in a natural manner is very difficult and needs to answer the following question: which is the principle underlying the naturalness of humans' movements and hence what are the implicit rules that, after several years of human movement observations, are part of Kinetography Laban? In Section 4 we propose possible answers to this question on the basis of recent neuroscience and biological studies. 


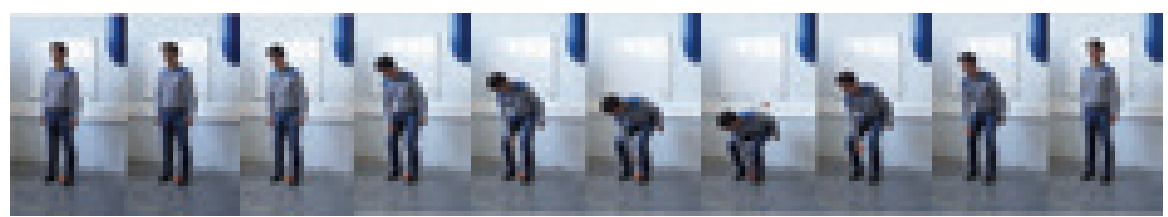

(a) Paolo takes the ball between its feet.

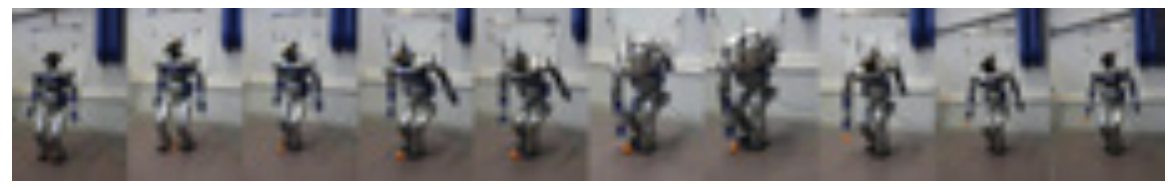

(b) HRP-2 takes the ball between its feet. To do that the robot has to step away. In this experiment, "stepping away" is not a specific software module. It is not a symbol. It is an integral part of the embodied action of taking. The entire motion is the output of the sole and unique software module "take".

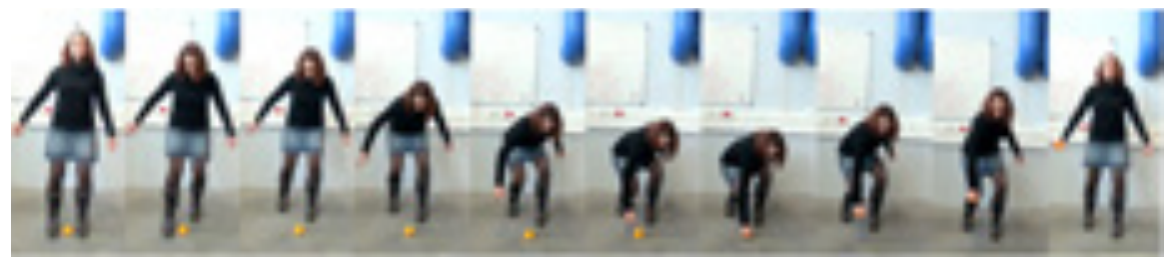

(c) Tiphaine is executing a motion by reading the notation in Fig. 3(c) which describes the movements of HRP-2 in Fig. 2(b) when it takes the ball. Tiphaine does not a priori know that she is taking a ball.

Fig. 2: To take the ball between its feet, contrarily to humans, the robot has to step away from the ball. This is due to the difference of morphologies between the human body and HRP-2 body.

\section{Robot programming and motion notation: a detailed example}

Let us consider the simple action of taking a ball. It might give rise to a complex motion involving the whole body and requiring the coordination of all body segments, if the ball is on the floor between the feet (see Fig. 2(a)). The legs have to naturally contribute to the action: "bending knees" becomes an integral part of the action "take the ball". In Fig. 2(b) the same action "take the ball" is programmed in the humanoid robot HRP-2. Based on a simple comparison between Fig. 2(a) and Fig. 2(b), it is straightforward to observe that humans and HRP-2 execute this action in different manners. Next subsections are dedicated to:

- describe how HRP-2 can be programmed in order to take the ball on the floor and explaining the differences between HRP-2 and humans engaged in the same action (Subsection 2.1), and 
- how the flexibility of the notation in describing human movements can be exploited to notate with different level of details (see Subsection 2.2), going from directly translating the sentence "take the ball on the floor between feet" to precisely describing the movements of all body segments.

\subsection{Robot programming: the Stack of Tasks}

One of the main feature of humanoid robots is their redundancy with respect to a task. This permits them to perform two or more tasks simultaneously, as e.g. taking an object with the right hand while putting the left one in touch with a fixed object to help legs ensure stability of the whole body. Of course, the robot can accomplish several tasks at the same time if and only if all of them are compatible each other: to check it, a simple idea is to put them in order.

The task-function approach, ${ }^{10}$ or operational-space approach, ${ }^{11}$ provides the mathematical framework to describe the hierarchy of tasks in terms of specific output functions, each one being a function from the configuration space to an arbitrary task space. At each time step of the integration process, a vector of the configuration space of the robot, tangent to the first task space, is selected. If the first task can be accomplished without using all the degrees of freedom of the robot, then a second task can be considered as soon it can be accomplished without interfering with the first one. By iterating this procedure, other tasks can be added until the whole set of degrees of freedom of the robot is completely exploited. The process can be iterated for other tasks so that a stack of tasks is obtained. ${ }^{9}$

Let us now use the stack of tasks method with the humanoid robot HRP-2 in order to execute the action "take the ball" as showed by snapshots in Fig. 2(a) where a human subject is executing it. The required movement is made more complicated by the fact that the ball is between the feet. However, the action is very simple and consists only in reaching the ball with the end-effector of the right arm (or the left one) of the robot and taking the ball, while maintaining the static equilibrium. As a consequence, the tasks to be ordered in the stack of tasks are basically two. The first one consists in maintaining the static equilibrium that, on a horizontal ground, is verified when the projection of the center of mass of the robot is inside its support polygon - the footprint in case of single support phase. The second one, which has a lower priority in the stack of tasks, consists in zeroing the error between the current position of the end-effector of the right arm and the position of ball on the floor. However, this is not enough for a humanoid robot. Indeed, other kinematic constraints are necessary to enforce the joint limits and avoid self-collision.

A solution to this problem has been provided. ${ }^{12}$ As shown in Fig. 2(b) the robot has to first step away from the ball and then grasp it. However, there is no dedicated module in charge of "stepping" and indeed, it is a direct consequence of "taking". The main reason why the robot step away from the ball can be found mainly in the kinematic constraint of avoiding self-collision. Moreover, by stepping away the robot reaches a position (the third snapshot in Fig. 2(b)) more comfortable for the 
robot, making easier the main task of maintaining the static equilibrium. The taking action is hence totally embedded in the body, allowing the legs to naturally contribute to the action. In this example, "taking" is an embodied action.

\subsection{Motion notation: the Kinetography Laban}

The Kinetograhy Laban allows to write down not only dances but all kinds of human movements observable by human eyes. The utility of the Kinetography, outside of choreographic field, is mainly based on analyzing human actions. The Laban system describes a movement by four factors: space (by direction and level), duration of the movement, beginning and end of the movement, and body parts. A complex action can be segmented according to these categorizations.

One of the basic element Laban notators use to write down a movement is the direction symbol. These symbols reflect a common approach to movement description in terms of spatial directions into which the part of the body move with the aim of reaching a given position. ${ }^{13}$ The pathway taken is less important than the final destination. The Kinetography Laban is a movement notation because the symbols represent changes. As a consequence, an absence of movements is represented by an absence of symbols.

The directions in space emanate from a central point called place. It is represented by a rectangle. Directions and levels are computed from this point. There are 8 main directions and 3 shading levels to form 27 principal directions represented by modifications of the rectangle and by shading of each symbol. On their own the direction symbols state only information concerning the element of direction. ${ }^{13}$ Only when they are placed in the appropriate column of the vertical staff it is possible to know which part of the body moves. In particular, for movements of the limbs direction and level are determined by the spatial relationship of the free-end (extremity of the limb) to the base (point of attachment). A line drawn between the extremity and the point of attachment indicates to which direction the limb has to move. The end can move with respect to the point of attachment, which is the point from which all the directions and levels specified by direction symbols radiate. For example, the whole arm is attached to the body by the shoulder. The shoulder is the point from which all directions and levels radiate. The whole arm can move with respect to the shoulder in order to place the hand, which is considered in this case as the free-end point of the arm.

We notated the action of "taking the ball" in three manners. The notation of Fig. 3(a) is one of the simplest way among three to describe the action. This notation does not mention how to take the ball in detail, but it indicates only a starting position (standing), arms positions at the beginning and the end of the action (the arms are stretched out along the body), the position of the ball at the beginning (it means that the ball is on the floor between the feet.), and the right hand grasps the ball at a given moment. The only information included in the notation on the way 


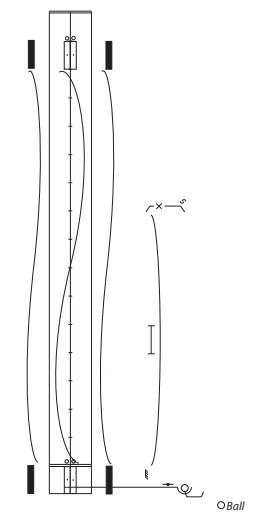
(a) Notation of the action "Take the ball" by using the Kinetography Laban.
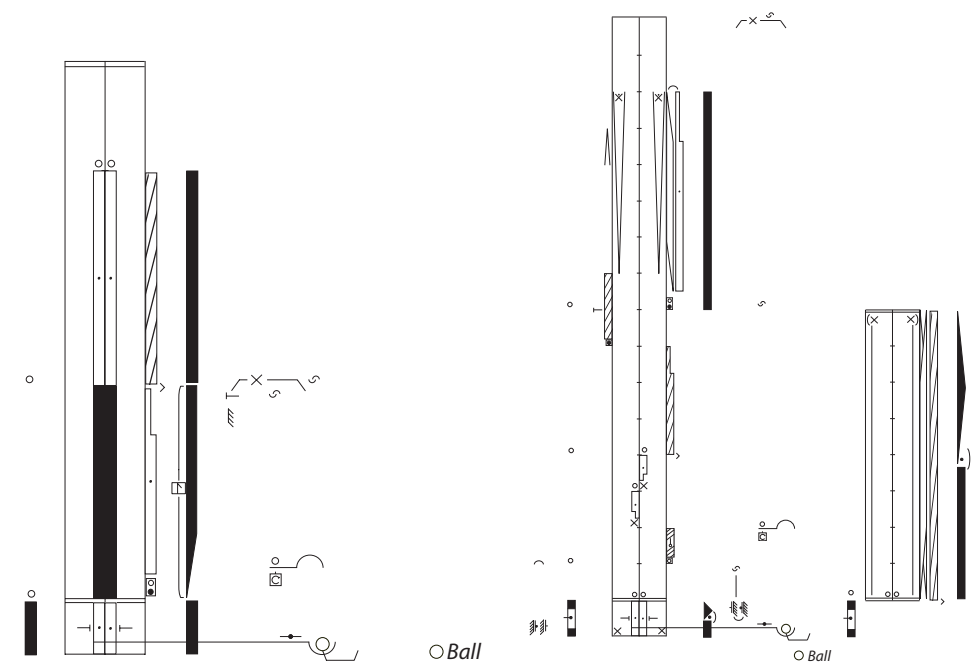

(b) The detailed description of the movement in Fig. 2(a) by using the Kinetography Laban.

(c) The detailed description of the movement in Fig. 2(b) by using the Kinetography Laban.

Fig. 3: Different Laban scores describing the motions motivated by the action of "Take the ball". The figures may appear as obscure for readers not aware about Laban notation details. Their purpose is mainly illustrative to show that differences appear. Moreover, the presence of a symbol modeling the ball argues that the notation not only deals with the movement of the human body parts, but also with the movement of the ball. 
of taking the ball is that the right hand follow a direct path to reach the ball. This is exactly the same information that is given to the robot via its programming system.

When we asked Paolo to take the ball without giving any constraints, he takes the ball without changing his feet positions. He just bends his knees and his hand grasps the ball. "Bending knees" is not explicitly expressed by the Laban score in Fig. 2(a).

The score depicted in Fig. 3(b) is the notation of detailed Paolo's movement. The notation describes his manner to take the ball with many details. It includes the way to reach the floor (e.g., rotation of the torso), the way to grasp (e.g. the choice of the right hand), the direction of the gaze, and a motion timing.

Fig. 3 is a notation of the whole movement of HRP-2 robot. It includes exactly the same level of details as the score in Fig. 3(b).

All the three scores differ. This is not a weakness of the notation system, but a strength. Indeed these three scores illustrate how the detail of an action can be described according to an intention of the notator. A same action may be noted with different levels of details according to the purpose of the notation, including what the notator wants to transmit to the performer and who is the reader of the score.

\section{The Kinetography Laban as robot programming tool}

In this section, we show how the Laban score and in particular its 27 direction symbols can be translated in the framework of the Stack of Tasks. We will achieve this goal by a worked-out example where the simulated humanoid platform Romeo has to execute a kind of hip-hop dance, also known as "Tutting Dance", that involves only the upper body, especially arms and hands, to create geometric shapes and movements.

\subsection{The Tutting Dance: from dancer movements to the Laban score}

The Laban score of the Tutting Dance sequence is shown in Fig. 4. The postures of the subject at each step of the Laban score are depicted in Fig. 5.

The score contains 9 columns. Each column is associated to a body part. The symmetrical stuff represents the symmetry of the body. The central columns on both sides of the main vertical axis represents the support of the body. Then the second columns are dedicated to the movement notation of the right and left legs. The third column, immediately outside the staff, are used for the torso and its parts. Tutting Dance mainly concerns the arms and the hands. This is why the second and

$\mathrm{i}$ The original version of the Tutting dance can be found at the following link https://www.youtube.com/watch?v=082Akz8hGLY 
third columns are free from any symbol, while the support columns contain only two small circles, which represent the "hold weight sign", just after the double line which indicates the start of the movement. In this case, these symbols indicate that the actor has to maintain the standing posture and the weight on the feet.

The forth columns on the right and on the left of the body columns correspond to the right and left arm gestures, respectively. The fifth columns correspond to both forearms gestures, the sixth to both upper arms gestures, the seventh to the right and left hand gestures, the eighth to the back and palm of the right and left hands. Finally, the last columns on the right and on the left correspond to the edge of fingers. The duration of the sequence is decomposed into 16 intervals according to our movement segmentation. Moreover, based on our observations, we deduced that no movement is faster or slower than others. As a consequence, in the Laban score the direction symbols have the same length meaning that all the movements have the same duration.

A detailed description of each movement is here reported:

0. This is a starting point. An actor is standing. Both arms are stretched out along the body.

1. The right arm goes to the right-middle direction.

2. The left arm goes to the left-middle direction.

3. The right upper arm goes to the forward-middle. The right forearm goes to the left-middle direction.

4. The left upper arm goes to the forward-middle direction. The left forearm goes to the right-middle direction.

5. The left forearm goes to the forward-middle direction.

6. The right forearm goes to the forward-middle direction.

7. The left hand goes to down.

8. The right hand goes to up.

9. Both forearms go to up. During this movement, both hands maintain their configurations with respect to the forearms.

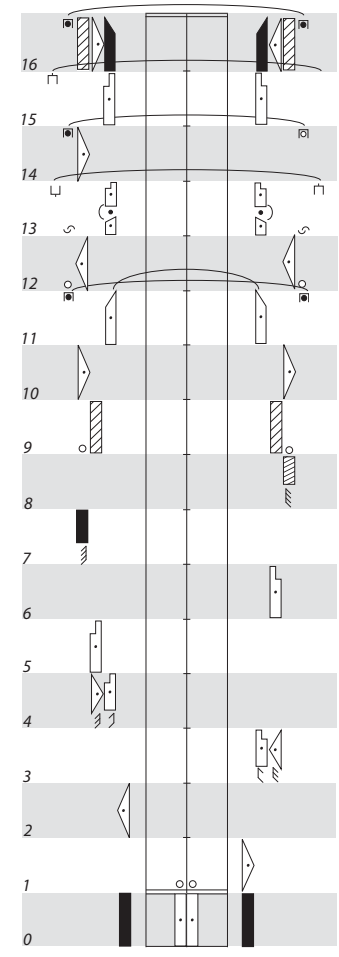

Fig. 4: Laban score for the Tutting dance

10. Both hands go to the right-middle direction.

11. The right upper arm goes to the left-forward-middle direction. The left upper arm goes to the right-forward-middle direction. Both upper arms are in contact. The left and right palms are also in contact.

12. The right and left hands while maintaining the contact, changed their direction to the left-middle.

13. Cancel the hold of the contact. The right upper arm goes to the intermediary direction between forward-middle and left-forward-middle. The left upper arm 
goes to the intermediary direction between forward-middle and right-forwardmiddle. As a consequence, the palms separate. The edge of the right finger touches the edge of the left hand.

14. The left hand goes to the right-middle direction. The left palm is in contact with the back of the right hand.

15. Both upper arms go to the forward-middle direction. The edge of both fingers are in contact.

16. The upper right arm goes to the right-forward-low direction. The right forearm goes to the left-middle direction. The upper left arm goes to the left-forwardmiddle direction. The left forearm goes to the right-middle direction. The hands go to up. Both palms are in contact.

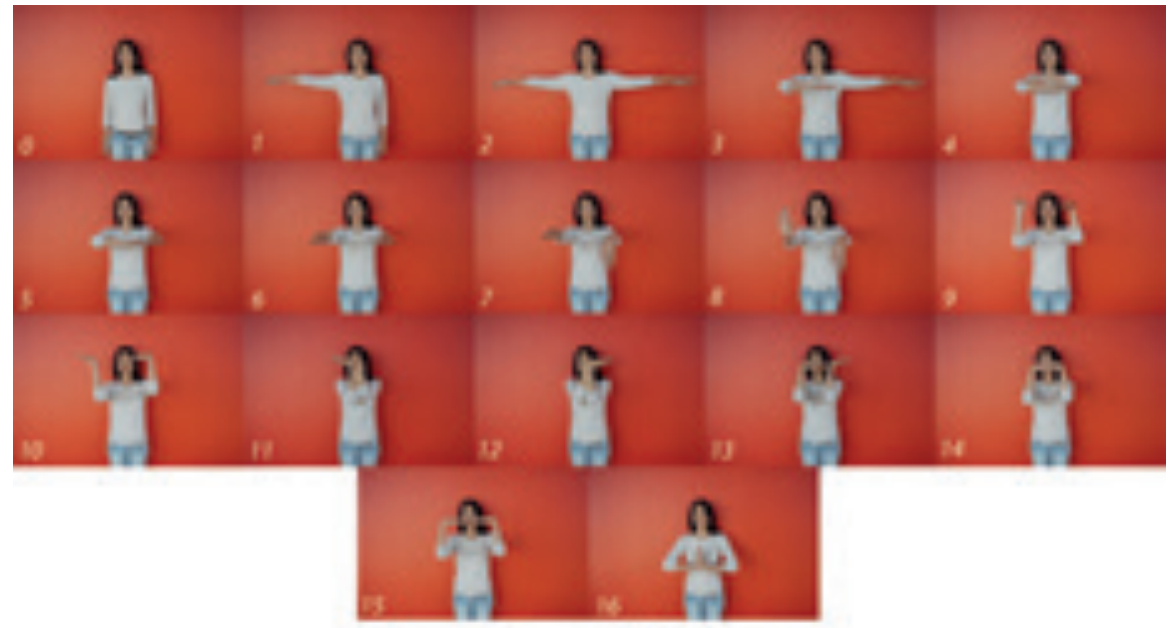

Fig. 5: Tutting dance

\subsection{From the Laban score to Romeo movements}

Starting from the Laban score of the previous section (see Fig. 4), the Tutting Dance is now translated in the SoT so as to generate suitable control signals for the motors to execute the movements in the humanoid robot Romeo.

We have seen that one of the basic element Laban notators use to describe a movement is the direction symbol. The Tutting dance we have chosen leads itself very well to this type of approach due to its geometric shapes and movements.

The 27 principal direction symbols used to describe the Tutting Dance in Fig. 4 are the starting point to translate the Laban score in the SoT. In other words, depending on the current configuration and the body part which the symbol refers to, each 
principal direction symbols, and hence the main directions and levels, are translated as reference positions in space around the point of attachment. Each reference position is defined by an homogeneous transformation matrix that specifies both desired position and orientation of a reference frame attached at the free-end point of the movable part of the Romeo. Based on the current position of the body part and the desired one specified by one of the principal direction symbol, a task function is defined as the error in terms of both rotation and translation between the current position in space of the reference frame attached to the free end and the desired one. The SoT is then used to determine suitable control signals for the motor of the robot such that this error becomes zero, while guaranteeing at the same time other tasks. These signals correspond to reference velocities or accelerations, the last ones providing a smoother movement for the robot. The first and most important task in humanoid robots is to maintain the static equilibrium. On a horizontal ground, the static equilibrium holds as soon as the projection of the center of mass of the robot is inside its support polygon - the footprint in case of single support phase. For the Tutting dance there is no displacement of the entire body (i.e. the so-called weight in Laban terminology and the so-called root of the kinematic tree in the robotics terminology) and indeed, in the support column of the Laban score, there are no symbols apart from the ones (small circles) that specify the maintenance of the starting feet positions. As a consequence, in the SoT, the first task, called "Weight on the feet" in Fig. 6, consists in guaranteeing the weight of the body rests on the feet.

We have seen that the absence of movements is represented by the absence of symbols. To include this rule in the humanoid robot Romeo, the last task in the stack, called "Reference posture" in Fig. 6, consists in limiting the "distance" from a reference configuration that can be considered as the natural standing position for humans. Just before this task, so at higher priority, a task, called "Torso fixed" in Fig. 6 is added in order to account for the lack of symbol in the body columns. Between the first task "Weight on the feet" and the task "Centre of Mass", other tasks representing the gesture symbols that follow one another in the Laban score will be added as represented in Fig. 6.

Another rule of the Kinetography Laban is that, after each symbol, until a new symbol does not involve the same part of the body (e.g. the whole arm), or a subpart of it (e.g. the forearm), the previous symbol holds. For this reason, in the stack of task some tasks change priority before disappearing from the stack: for example, referring to Fig. 6, task "Right Arm - middle/right" has a lower priority in SoT 2 with respect to SoT 3.

The final result of the implementation of the Tutting dance in Romeo is shown in Fig. 7. Next section is dedicated to show the main differences between Romeo and human movements. 


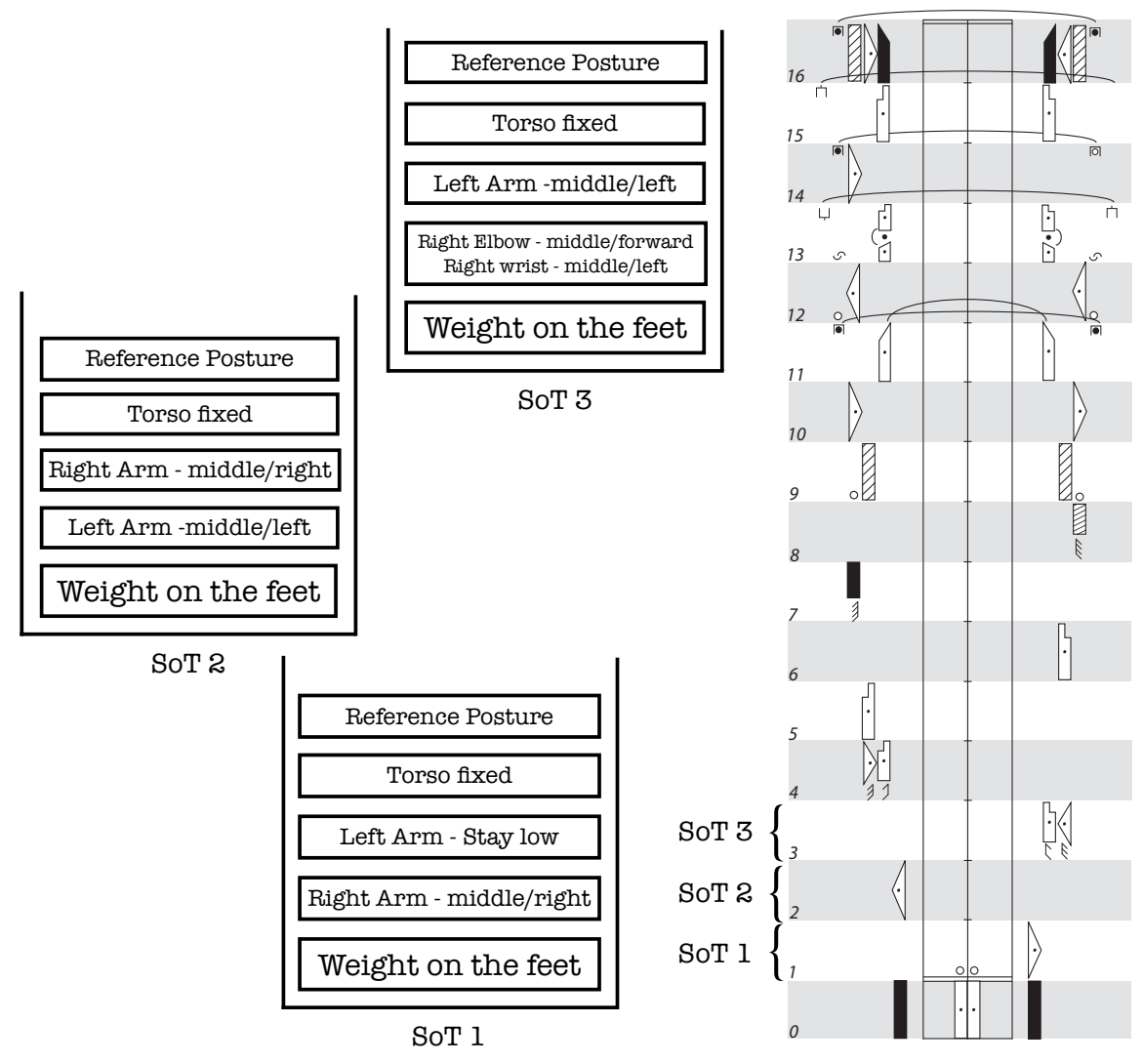

Fig. 6: Stack of tasks for the first tree step of the Laban score.

\section{Discussion}

In this section, we will first discuss on the obtained results, by comparing the movement of Romeo with the movement of Naoko, also by means of the Kinetography Laban. The second subsection is dedicated to describe some rules of the Kinetography Laban about how movements should be executed, and plausible origins of these rules.

\subsection{Comparison between human and Romeo movements}

In the previous section, the Laban score of a simple example of Tutting Dance has been translated, by using the SoT, in suitable control signals for the motor of the simulated humanoid robot Romeo. In Fig. 7, the snapshots corresponding to the La- 


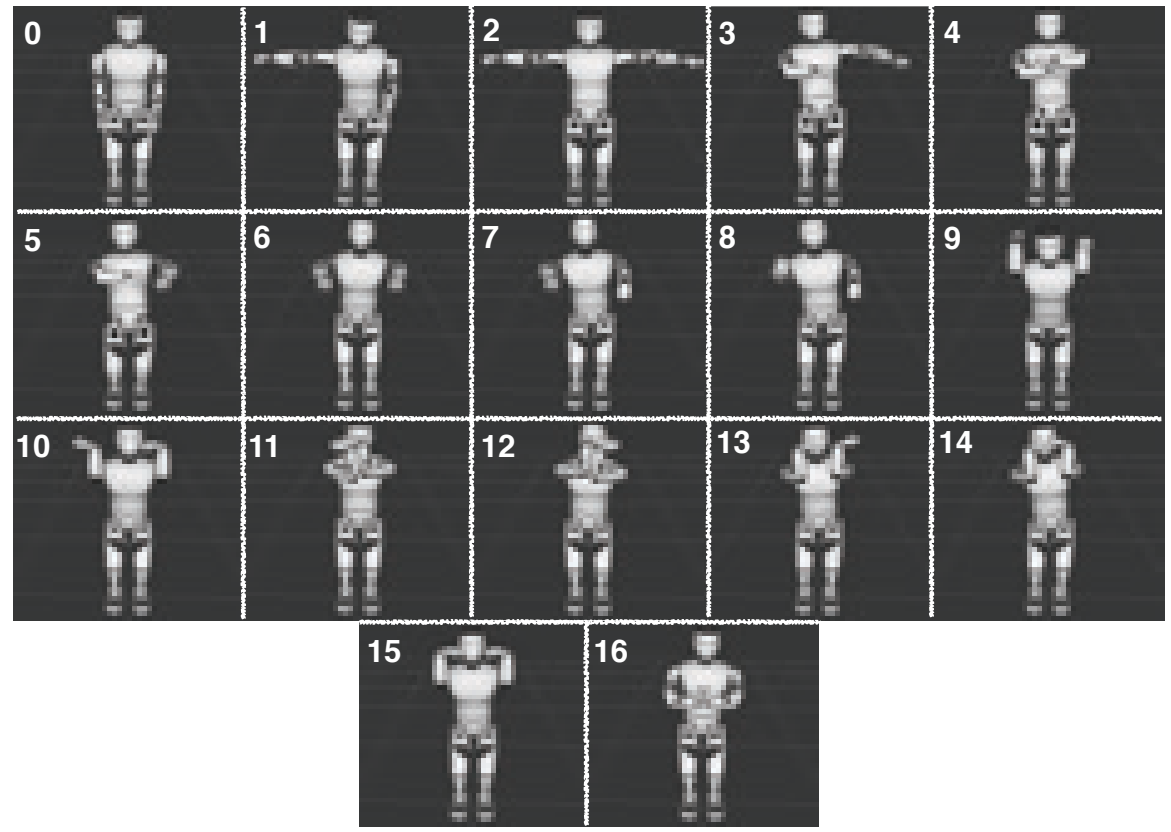

Fig. 7: Tutting dance realized by Romeo. The numbering of each snapshot corresponds to the numbering in the Laban score of Fig. 4.

ban score in Fig. 5 is reported. Apart from the standing posture of Romeo which is slightly different from humans (the legs are slightly bent, as most humanoid robots), there are not significant differences w.r.t. the snapshots in Fig. 5. However, in snapshot 3 of Fig. 7, the left arm of Romeo is bent while the left arm of Naoko is straight (see snapshot 3 of Fig. 5). Moreover, mainly in the asymmetric postures, the torso, the pelvis and shoulders of Romeo are not exactly at the same configurations of Naoko at the end of each step.

All these different configurations at the end of each step are a direct consequence of the movement executed by Romeo while progressing in the Laban score. Indeed, by using the SoT to generate the movements, each motor of Romeo is controlled such that the free end of a body segment moves along a straight line passing from both the initial position and the final one, as represented in Fig. 8. The movement corresponds to the step 6 in the Laban score. The free end of the right forearm should move following a circular arc centered at the elbow, i.e. performing a peripheral movement, and the rest of the body should remain fixed. Based on the snapshots in Fig. 8 this is not the case, as it is also pointed out in Fig. 9.

This characteristic gives hence rise to undesirable movements of the body. In Fig. 9(a), the movement corresponding to the first step in the laban score and to SoT 1 in Fig. 6 is shown. All these differences can be also appreciated by notating the Romeo movement with the Kinetography Laban. In Fig. 10 a comparison between 


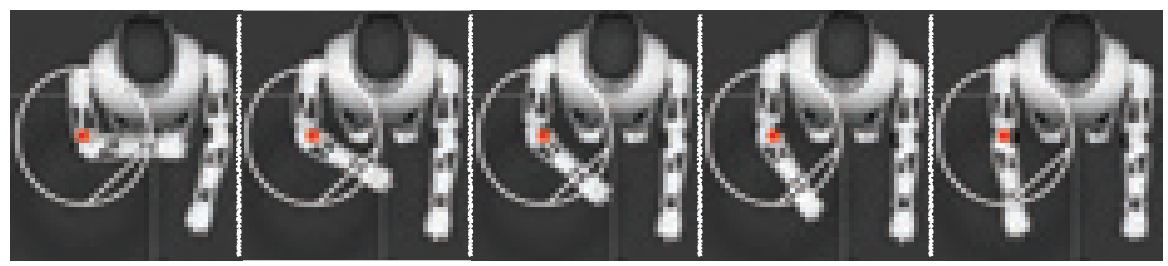

Fig. 8: Referring to Fig. 4, this is a sequence of snapshots for the movement at step 6 in the Laban score of Fig. 4. The free end of the right arm moves along a straight line between the starting position to the final one. Hence, the elbow (red point) does not remain at a fixed position in space. This makes the difference with the original motion performed by Naoko.

the Laban scores of Romeo's and dancer's movement during the first 6 steps is reported. Notice that now, in the Laban score of Romeo (see Fig. 10(b)), the columns for the body gestures is not free of symbols and signs. Moreover, on the side of each direction symbol, a new sign is notated, representing a description of the path that the free end of the arm is now executing - basically, a straight line. In Fig. 9(b), snapshots corresponding to the movement from step 2 to step 3 of the Laban score is also reported. In this case, the movement of the whole body is much more noticeable.

Finally, even if the Laban score in Fig. 10(a) is a so called legato movement, that is with no separation between direction symbols and hence with no interruption in the continuity of the movement, for Romeo, sometimes there is a sort of overlapping between consecutive direction symbols and hence movements that indeed should be consecutive. This means that still before the previous movement is finished, the next one is already started (see Fig. 10(b) between steps 3 and 4).

\subsection{On the naturalness of movements}

As already said in previous section, on their own the direction symbols state only information concerning the element of direction. Only when they are placed in the appropriate column of the vertical staff it is possible to know which part of the body has moved. Moreover, depending on the actual configuration of the body part, due to implicit rules of the Kinetography Laban created and based on the naturalness of human movements, information on the path of the free end of that part of the body can be also achieved.

In using the 27 principal directions around the body, symbols that correspond to adjacent points in space are considered to be at a first-degree distance from one another (see Fig. 11). For example, if the arm moves from forward middle to the adjacent right front diagonal point, this is a first-degree distance. In this case, the free end of the arm, i.e. the hand, describes an arc of circle whose center is the shoulder 

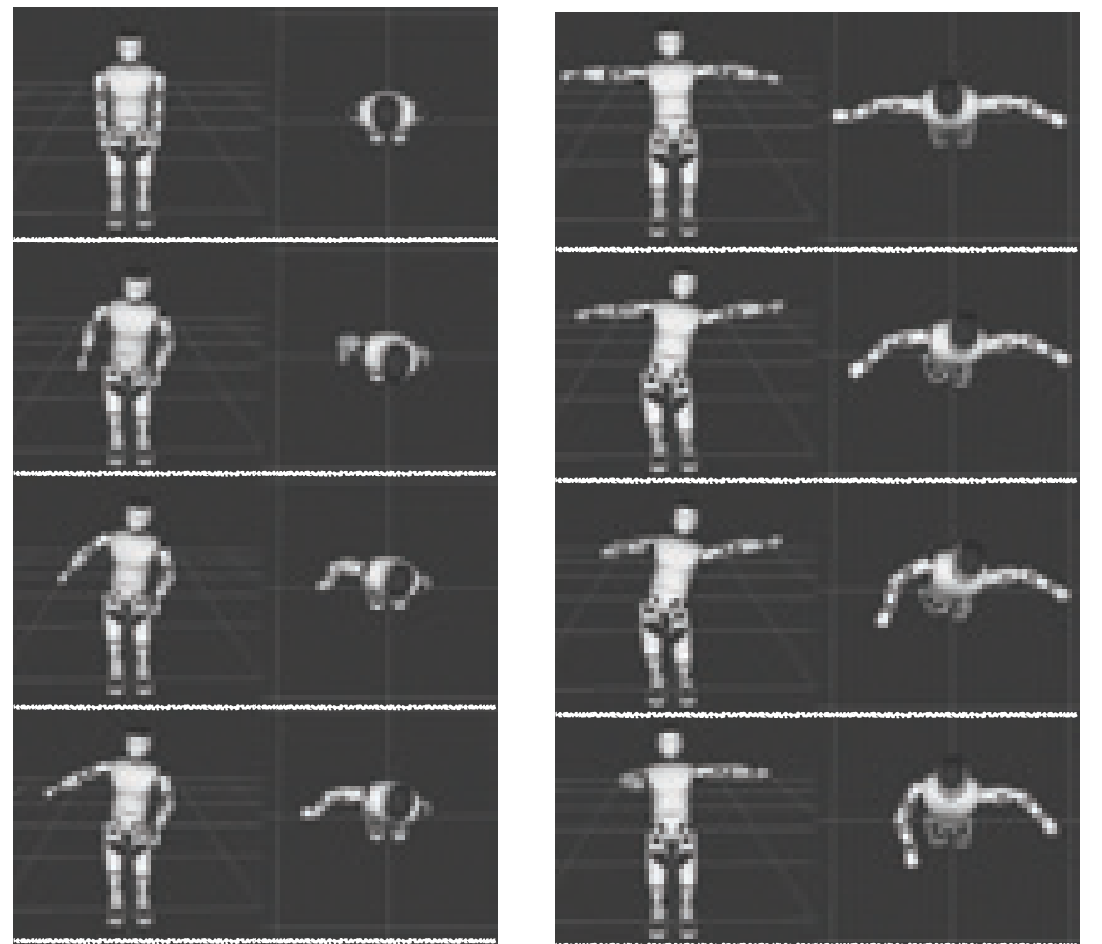

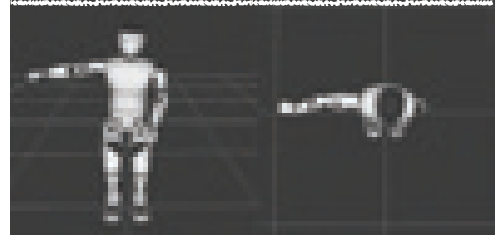

(a) The movement of Romeo at step 1 in Fig. 4

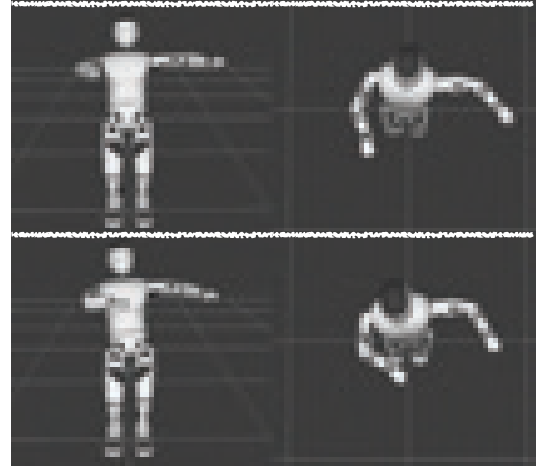

(b) The movement of Romeo at step 3 in Fig. 4

Fig. 9: Two movements executed by Romeo. All movements describe straight line paths for the free end of the arms. This gives rise to undesirable movements of the body.

moves on the surface of the sphere. This is the so called peripheral movement in Laban system. ${ }^{14}$ All movements between first-degree distance points will produce this path. In case of the arm moves from forward middle to side (right or left) middle, we have a second-degree distance (see Fig. 11) and the hand describes a quarter of circle whose center is the shoulder, it is also called peripheral movement. All movements between second-degree points have to be performed without any special flexion of the arm unless otherwise specified with the addition of a particular sign, as e.g. the straight path sign. 


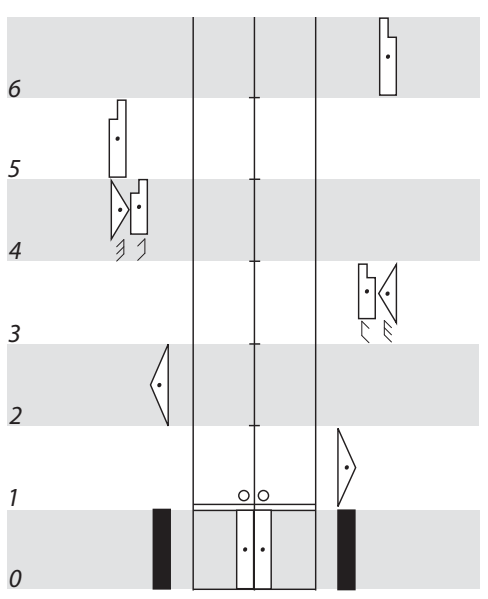

(a) Laban score for a dancer

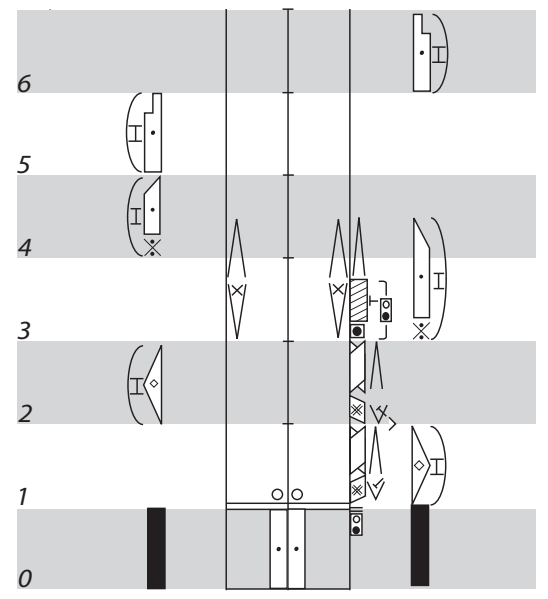

(b) Laban score for Romeo

Fig. 10: A comparison between the Laban score of the Tutting Dance executed by a dancer and Romeo.

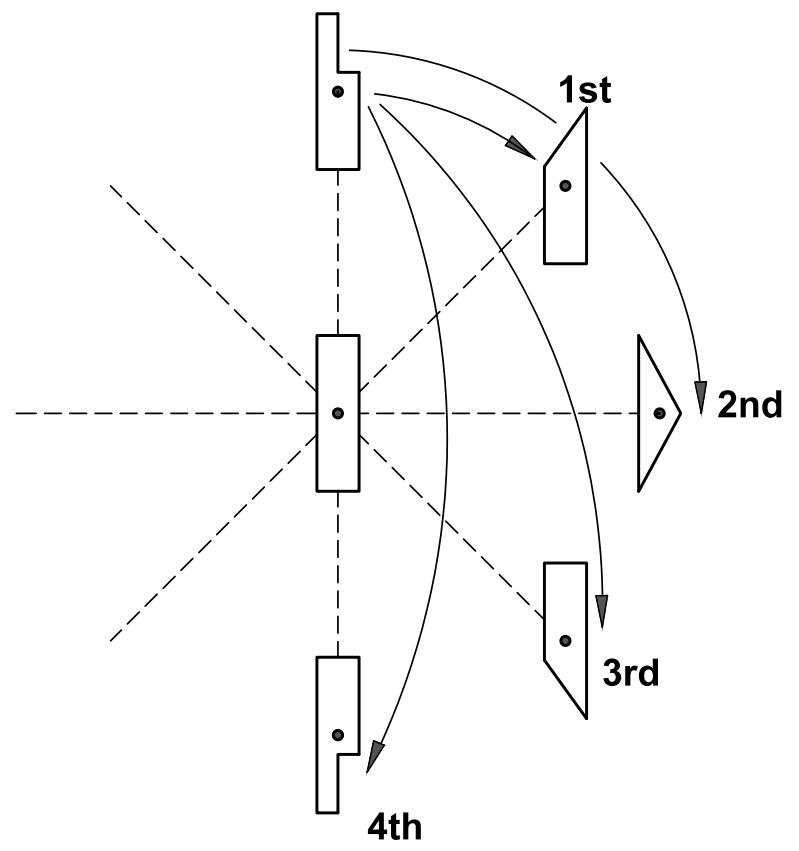

Fig. 11: Degree of distance between points.

If two points are at third-degree distance, the free end of the limbs moves along a trajectory closed to the body, and not peripheral path. In this movement, the arm is 
slightly bent, takes a path between periphery and center ("in place"). This is called intermediate situation or transversal movement. ${ }^{14}$

Finally, diametrically opposite points are considered to be at a fourth-degree distance. For example if the arm moves from forward middle to the extreme opposite direction backward middle. The arm comes back "in place" then extends again to outside. These type of movements are called central movement in Laban system. ${ }^{14}$

The control laws used to move the arms of Romeo do not contain all this variety of movements. Independently from the distance of points in space, Romeo always performs a straight line path, with noticeable loss in naturalness. Indeed, to perform that movement, other undesirable and unnatural body movements are necessary. Of course, to solve this problem, an ad-doc control law that moves the hand along peripheral, central, or transversal movement might be determined.

On the other hand, the implicit rule of the notation comes from the several years of observations and it is based on the naturalness of human movements, also induced by the mechanical structure of the body. As often conjectured in robotics, an optimality principle might underline human movements. It would be hence interesting to understand what is this principle, to express it in a suitable mathematical manner and than to use it to determine the control laws for the humanoid robot Romeo, by using tools as the optimal control theory.

The main problem in translating the Laban score in humanoid robots is hence to obtain the continuous movement that resemble the human one. To this end, several biological studies show principles that explain human movements. ${ }^{15-18}$ In particular, considering only arm movements in an horizontal plane, the recorded trajectories are well explained in terms of minimum jerk. ${ }^{15}$ On the other hand, when arms move on a vertical plane (in this case the force of gravity plays a fundamental role, making the movement asymmetric), the recorded trajectories are well explained in terms of the minimum sum of jerk and energy. ${ }^{18}$

\section{Conclusion}

What are the lessons learned from this attempt to make use of Kinetography Laban in humanoid robot programming?

Three study years are necessary to be graduated in Laban notation. Even for well trained notators, scoring simple movements as the ones described in this chapter is time consuming. We have seen that a same action may be notated with different levels of details. Indeed Laban notation addresses the movement more than the action itself. Two scores may symbolize the action of "taking a ball" according to the importance one gives to the manner to take a ball. Both scores are complementary; they are not opposite. For a roboticist, making use of Laban notation to program a humanoid robot requires either to have been trained for several months, or to work with a notator. At the very end, the notator describes the movements in the physical space and the roboticist translates the score symbols in terms of Jacobian inversion. More the score is detailed, more the inversion task is tedious. This makes the ques- 
tion of automated translation a critical issue. In spite of few tentatives referenced in the introduction of this paper, the question remains largely open, as soon as the objective is to account for all dimensions and richness of the Kinetography Laban notation.

Human body and humanoid robot body differ. Retargeting a human movement on a digital artifact is a well known issue in computer graphics. ${ }^{19}$ This is the same in humanoid robotics. We have seen that HRP-2 robot cannot take the ball as Paolo did. This is due to the fact that the body of HRP-2 prevents the robot to put its hand between its feet without stepping away. To take the ball on the floor, HRP2 robot may benefit from the score in Fig. 3(b) but not from the score in Fig. 3(c). Human motion notations are all based on the kinematic structure of the human body. Adapting the notation to another structure is certainly possible, but it is a challenge by itself.

Finally we have seen that the question of the naturalness of a movement is another critical issue. Laban notators benefit from a lot of implicit knowledge that ground the Kinetography Laban. The issue has been clearly revealed in Section 4.2. The rules to move the hand in a given direction have been defined and described on the basis of a long experience in observing human movements. Movement notators target a movement description. They are not a priori interested by causality principles, i.e. by the origin of the movement. The origin of the movement takes place in the muscle control space. However, it is not necessary to tell a dancer what muscles he/she has to activate to perform a desired movement. Muscle activation is an unconscious process. With the fundamental problem of inverting actions expressed in the physical space into motor controls, roboticist have to face the causality principle. This is why, like neurophysiologists, roboticists try to exhibit general movement laws to explore plausible causality principles.

To summarize the experience gained with the worked-out examples described in this paper, we can say that dance notation and robot programming pursue two different goals in two different spaces. The goal of the dance notator is to describe the qualities of the movement as wished by the choreographer while the roboticist is a priori concerned by the action to be performed, better than by the motion that fulfills the action. However we have seen that there is an interest for roboticists to consider dance notation as a way to better explore the relationship between action and motion. Symbolical and computational foundations of both motion and action concepts, as complementary developed by dance notation practitioners and roboticists respectively, deserve to be deeper explored.

Acknowledgements This work is supported by ERC-ADG project 340050 Actanthrope. Authors thank Noëlle Simonet, professor of the Kinetography Laban at CNMDP (Conservatoire National de Musique et de Danse de Paris), for reviewing the Laban scores, and Tiphaine Jahier, dancer and Laban notator, for her participation to read notations and perform actions. 


\section{References}

1. Ben Choi and Yanbing Chen. Humanoid motion description language, 2002.

2. Kanav Kahol, Priyamvada Tripathi, and Sethuraman Panchanathan. Automated gesture segmentation from dance sequences. In Automatic Face and Gesture Recognition, 2004. Proceedings. Sixth IEEE International Conference on, pages 883-888. IEEE, 2004.

3. Kanav Kahol, Priyamvada Tripathi, Sethuraman Panchanathan, and Thanassis Rikakis. Gesture segmentation in complex motion sequences. In Proceedings IEEE International Conference on Image Processing, pages 105-108, 2003.

4. A. Hutchinson Guest. Choreo-Graphics, A comparison of Dance Notation Systems From te Fifteeth Century to the Present. Gordon and Breach Science Publishers S.A., New York, 1989.

5. Malik Ghallab, Dana Nau, and Paolo Traverso. Automated planning: theory and practice. Morgan Kaufmann Publishers, Elsevier, 2004.

6. E. Yoshida, J.P. Laumond, C. Esteves, O. Kanoun, A. Mallet, and K. Sakaguchi T., Yokoi. Motion autonomy for humanoids, experiments on hrp-2 no. 14. Computer Animation and Virtual Worlds, 20(5-6), 2009.

7. Thierry Siméon, Jean-Paul Laumond, Juan Cortés, and Anis Sahbani. Manipulation Planning with Probabilistic Roadmaps. The International Journal of Robotics Research, 23(7-8):729746, August 2004.

8. D Sternad and S Schaal. Segmentation of endpoint trajectories does not imply segmented control. Experimental Brain Research, 124(1):118-136, 1999.

9. N. Mansard and F. Chaumette. Task sequencing for high-level sensor-based control. IEEE Transactions on Robotics, 23(1):60-72, Feb 2007.

10. Claude Samson, Bernard Espiau, and Michel Le Borgne. Robot Control: The Task Function Approach. Oxford University Press, 1991.

11. O. Khatib. A unified approach for motion and force control of robot manipulators: The operational space formulation. Robotics and Automation, IEEE Journal of, 3(1):43-53, February 1987.

12. Oussama Kanoun, Jean-Paul Laumond, and Eiichi Yoshida. Planning foot placements for a humanoid robot: A problem of inverse kinematics. International Journal of Robotics and Research, 30(4):476-485, Apr. 2011.

13. Ann Hutchinson Guest. Labanotation: the system of analyzing and recording movement. Psychology Press, 2005.

14. Albrecht Knust. Dictionnaire usuel de Cinétographie Laban (Labanotation). Ressouvenances, 2011.

15. T. Flash and N. Hogan. The Coordination of Arm Movements - an Experimentally Confirmed Mathematical-Model. Journal of Neuroscience, 5(7):1688-1703, 1985.

16. P J Stapley, Thierry Pozzo, G Cheron, and Alexander Grishin. Does the coordination between posture and movement during human whole-body reaching ensure center of mass stabilization? Experimental Brain Research, 129(1):134-146, October 1999.

17. Alessandra Sciutti, Laurent Demougeot, Bastien Berret, Simone Toma, Giulio Sandini, Charalambos Papaxanthis, and Thierry Pozzo. Visual gravity influences arm movement planning. Journal of Neurophysiology, 107(12):3433-3445, June 2012.

18. Jérémie Gaveau, Bastien Berret, Laurent Demougeot, Luciano Fadiga, Thierry Pozzo, and Charalambos Papaxanthis. Energy-related optimal control accounts for gravitational load: comparing shoulder, elbow, and wrist rotations. Journal of Neurophysiology, 111(1):4-16, January 2014.

19. R. Parent. Computer Animation: Algorithms and Techniques. Morgan Kaufmann Publishers, Elsevier, 2001. 